\title{
Estudio seroepidemiológico de la toxoplasmosis en niños de áreas de riesgo de la ciudad de Río Cuarto. Córdoba. Argentina
}

\author{
ALICIA E. CHIARETTA*, ANA M SBAFFO*, ANDREA L. CRISTOFOLINI* y MARIANO D. MOLINA*
}

\author{
SEROEPIDEMIOLOGICAL STUDY OF THE TOXOPLASMOSIS IN CHILDREN \\ OF AN AREA OF RISK OF RIO CUARTO CITY. CÓRDOBA. ARGENTINA
}

This research was made during the years 1999-2001, within an interinstitutional programme between Universidad Nacional de Rio Cuarto (UNRC) and the Town Hall of the same city where researchers worked together with health care personnel placed in the research area. The analysed area is situated within the city urban radio and it expands on both margins of the rio Cuarto. The main objective of the study was to determine the seroprevalence to Toxoplasma gondii in infant and young children population ranging from 0 to 15 years old living in risky areas in Rio Cuarto city and to analyse and interpret those variables that interact in the presentation of this zoonosis.. It was of cross section, estimating the sampling in 257 children, being carried out a screening of 1/16 by means of the technique of Indirect Immunofluorescence. Epidemiological surveys were made which let the researchers determine the risk degree of some geo-bio-enviromental factors involved in infestation. A seroprevalence of $51.75 \%$ was found. . No significant differences were found according to sex; an increase in prevalence was found with respect to age; there was neither undercooked meat nor insufficiently cleaned garden vegetables consumption. Coexistence with cats and/or dogs, especially in soil surrounded dwellings (gardens, corrals, orchards, henhouses) was defined as a risk factor. Therefore, contact with $\boldsymbol{T}$. gondii oocysts contaminated environment has been determined as the most important transmission source for children coming from the researched area.

Key words: Prevalence, Toxoplasma gondii, Survey, Zoonosis.

\section{INTRODUCCIÓN}

La toxoplasmosis, es la zoonosis parasitaria más difundida en la naturaleza ${ }^{1}$. Se ha encontrado en todos los continentes, tanto en poblaciones humanas como en más de 330 especies de animales domésticos y/o silvestres ${ }^{2}$. El Toxo- plasma gondii, su agente causal, si bien se encuentra en muchas especies, es sólo en los felinos en donde se producen formas sexuadas capaces de producir ooquistes contaminantes del ambiente ${ }^{3}$.

Cuadros clínicos en personas ocurre por regla general en forma muy esporádica y son de baja

* Departamento de Patología Animal, Facultad de Agronomía y Veterinaria, Universidad Nacional de Río Cuarto, Ruta 36 Km 601, Río Cuarto, Argentina.

Financiado por SeCyT. Universidad Nacional de Río Cuarto. Argentina. 
incidencia. Su importancia en Salud Pública reside sobre todo en la gravedad de la infección congénita y sus secuelas ${ }^{4}$. La tasa de reactores se acrecienta con la edad al aumentar las oportunidades de adquirir la infección, lo que depende de varios factores, entre otros, el nivel social y cultural de la población, la higiene ambiental, la convivencia con animales domésticos (perros-gatos) y los hábitos alimenticios en cuento al consumo de carne cruda o insuficientemente cocida ${ }^{6}$.

En distintas localidades de Argentina se han determinado sus prevalencias y difusión, tanto en humanos como en animales ${ }^{5}$. En Río Cuarto el único antecedente que existe, es un estudio realizado en una población de 297 mujeres embarazadas, en el cual se obtuvo un $40,8 \%$ de seroreaccionantes a la técnica de Inmunofluorescencia Indirecta (IFI) ${ }^{7}$. El objetivo de la presente investigación será determinar la seroprevalencia de $T$. gondii en la población infantil de 0-15 años de edad, en áreas de riesgo de la ciudad de Río Cuarto. Además se analizará aquellas variables que podrían interactuar en la presentación de esta zoonosis y de ese modo conocer los mecanismos de transmisión y factores de riesgo determinantes en esta zona. Esta información epidemiológica, le permitirá a la Municipalidad el abordaje de la problemática existente, a través de programas específicos de control.

\section{MATERIAL Y MÉTODO}

Río Cuarto es una ciudad intermedia, agropecuaria; ubicada al sur de la provincia de Córdoba (Argentina), con alto índice de desocupación y pobreza focalizada ${ }^{8}$. Estas condiciones han originado 13 asentamientos poblacionales permanentes, que se ubican en las márgenes del río Cuarto; comprendiendo a 905 familias, constituidas por 3.615 personas, de las cuales $1.256(34,74 \%)$ corresponden al grupo etáreo entre 0-15 años ${ }^{9}$. Cuatro de estos asentamientos presentan la Tasa de Mortalidad Infantil (TMI) más elevada de la ciudad (6,73 $8,97 \%)^{10}$.

En estos sectores, las construcciones precarias, hacinamiento, saneamiento deficiente, entorno contaminante con formación de microbasurales, alimentación irregular, escaso nivel de instrucción de la población y falta de aplicación de medidas sanitarias y educativas de rigor; sumado a una gran cantidad de animales domésticos sueltos en la vía pública (equinos, porcinos, caninos y felinos), con un bajo porcentaje de desparasitación, vacunación, hábitos alimenticios en base a restos domiciliarios ${ }^{11} \mathrm{y}$ sin ningún método de control reproductivo, especialmente de la población canina ${ }^{10}$; generan condiciones ecológicas favorables para la difusión y transmisión de diferentes zoonosis, entre ellas la toxoplasmosis. El estudio, de corte transversal, se realizó durante los años 1999-2001: Se seleccionaron 8 de 10 Efectores de Salud (ES) ( se excluyen los $\mathrm{N}^{\circ} 3$ y 7), los cuales brindan ayuda asistencial gratuita a la mayoría de los individuos pertenecientes al área. Se calculó un el tamaño de la muestra (n) de 257 niños de 0 - 15 años de edad considerando una estimación de un $30 \%$ de prevalencia, una precisión del $5 \%$ y un nivel de confianza del $95 \%{ }^{12}$. Los niños se fraccionaron en cinco rangos etários, 0 - 3 años $(n=67) ; 4-6$ años $(n=73) ; 7$ - 9 años $(\mathrm{n}=45) ; 10-12$ años $(\mathrm{n}=17)$ y $13-15$ años $(\mathrm{n}=55)$.

A cada niño, se le extrajo por venipunción, una muestra de $5 \mathrm{ml}$ de sangre la que era enviada al laboratorio de Parasitología, perteneciente a la Facultad de Agronomía y Veterinaria de la Universidad Nacional de Río Cuarto, para su procesamiento. Las muestras fueron tomadas en el laboratorio central municipal, a los tres primeros pacientes de cada día, que no tuviesen lazos familiares entre sí, derivados desde los ES.

Se realizó un "screening" de $1 / 16$, determinando la presencia de anticuerpos IgG anti-toxoplasma, mediante $\mathrm{IFI}^{4,6}$.

Simultáneamente, la población muestreada fue evaluada mediante un cuestionario, el cual se hallaba organizado con preguntas cerradas, dicotómicas y de opciones múltiples. Con los datos obtenidos se creó una base (Software EpiInfo 2000 versión 1) donde fueron cargados además, los resultados serológicos con el objeto de interrelacionarlos y validarlos estadística y epidemiológicamente. Los mismos permitieron el estudio de 24 variables epidemiológicas, a través de su análisis estadístico mediante Chi Squares ( $\left.\mathrm{Chi}^{2}\right)$, P-values (P), Riesgo Relativo (RR) y Odd Ration (OR).

\section{RESULTADOS}

De los 257 niños, 133 ( 51,75\%) resultaron seropositivos a $T$. gondii, No se observaron 
diferencias estadísticamente significativas según sexo $(\mathrm{p}>0,05)$. La distribución de frecuencias halladas en cada grupo etareo se presenta en Tabla 1. De los niños del primer grupo de la Tabla 1, los menores de 1 año tenían un $23,08 \%$, los de 1 año un $60,00 \%$, los de 2 años un $35,71 \%$ y los de 3 años un $36,84 \%$ de infección.

Se procesaron sólo 249 encuestas ya que 8 casos no se encontraron habitantes en las viviendas o se negaron a responderla. Del total de niños estudiados, 126 convivían con gatos, 179 con perros, 97 con gatos y perros y 41 no poseían ningún animal doméstico. El estudio estadístico de las variables mencionadas, se muestra en las Tablas 2, 3 y 4 .

Un total de 240 viviendas tenían tierra en su peridomicilio, incluyendo gallineros, huertas, jardines y corrales; mientras que 9 poseían solamente cemento. De los primeros, 71 presentaron serología positiva a $T$. gondii y convivían con gatos; mientras que 66 resultaron negativos sin la presencia de esta mascota. Por otro lado, 101 de los positivos convivían con perros, mientras que 45 no lo hacían . El análisis estadístico de estos resultados, se presentan en las Tablas 5 y 6 .

En cuanto a la alimentación, en 198 hogares se consumían verduras con un adecuado lavado previo; mientras que tan sólo 4 no lo hacían. En el resto de los hogares no se ingería este tipo de alimento. Por otro lado, 208 familias consumían carne bien cocida, mientras que 41 la preferían semicocida. Cabe destacar que el 99,2\% de los individuos optaban por la carne vacuna.

\section{DISCUSIÓN}

La seroprevalencia de $51,78 \%$ encontrada en estos 257 niños, coincide con las cifras encontradas en países desarrollados como Francia, España, Canadá y Estados Unidos y en países latinoamericanos como Paraguay, Costa Rica, Panamá Brasil y México ${ }^{13-19}$. Argentina no escapa a esta situación, como lo indican los valores hallados en Tucumán ${ }^{20}$, Chaco $^{21}$, Buenos Aires $^{22}$, Córdoba ${ }^{23}$ y Santa $\mathrm{Fe}^{24}$; con prevalencias del $46 \%, 35 \%, 62 \%, 61 \%$ y $53 \%$, respectivamente.

La prevalencia en niñas fue ligeramente superior que la encontrada en niños; sin embargo, estas diferencias no fueron estadísticamente significativos $\left(\mathrm{Chi}^{2}=0,04 ; \mathrm{P}=0,836 ; \mathrm{RR}=0,97\right.$ $[0,77<\mathrm{RR}<1,24] ;$ OR $=0,95[0,55<\mathrm{OR}<$ $1,62]$ ); de tal manera que se puede inferir que, no existe asociación entre la probabilidad de contraer la infección y el sexo de los niños, lo que coincide con los resultados obtenidos por otros investigadores ${ }^{17-19}$.

Como se puede observar en la Tabla 1, Al relacionar los resultados serológicos con los

Tabla 1. Distribución de frecuencia de la toxoplasmosis en niños de acuerdo a rangos de edad

\begin{tabular}{rccc}
\hline $\begin{array}{c}\text { Rango } \\
\text { Etareo } \\
\text { (Años) }\end{array}$ & $\begin{array}{c}\text { Número } \\
\text { Estudiado }\end{array}$ & $\begin{array}{c}\text { Serología } \\
\text { Positiva }\end{array}$ & Porcentaje \\
\hline $0-03$ & 67 & 38 & 41,79 \\
$04-06$ & 73 & 37 & 50,63 \\
$07-09$ & 45 & 24 & 53,33 \\
$10-12$ & 17 & 10 & 58,82 \\
$13-15$ & 55 & 34 & 61,82 \\
\hline
\end{tabular}

Tabla 2. Serología anti Toxoplasma gondii y tenencia de gatos

\begin{tabular}{lccc}
\hline & Positivos & Negativos & Total \\
\hline Tenencia & 76 & 50 & 126 \\
Ausencia & 55 & 68 & 123 \\
Total & 131 & 123 & 249 \\
\hline
\end{tabular}

Tabla 3. Serología anti Toxoplasma gondii y tenencia de perros

\begin{tabular}{lccr}
\hline & \multicolumn{2}{c}{ Serología } & \\
& Positivos & Negativos & Total \\
\hline Tenencia & 107 & 72 & 179 \\
Ausencia & 24 & 46 & 70 \\
Total & 131 & 118 & 249 \\
\hline
\end{tabular}


diferentes rangos etarios, se observó que la prevalencia a $T$. gondii, se acrecienta con la edad. Esto podría deberse a que a mayor edad, el individuo se encuentra más expuesto a las diferentes fuentes de transmisión del parásito ${ }^{18,19}$.

En el rango de 0 - 3 años de edad, se pudo advertir que los niños de 1 año presentaban la mayor prevalencia $(60 \%)$. Esta situación puede deberse a que la inteligencia del niño en esa edad se caracteriza fundamentalmente por adquirir una mayor madurez psicomotora y el alejamiento físico de su madre, lo que le permite explorar el exterior, el gateo y el desarrollo de una primacía de lo sensorio-motor lo que hace que manipule objetos contaminados y pueda llevándoselos a la boca. De este modo, dichos comportamientos propios de la edad, estarían favoreciendo el contacto entre el niño y el medio ambiente contaminado con ooquistes de $T$. gondii ${ }^{19,25}$.

$\mathrm{Al}$ correlacionar la variable "tenencia de gatos", con la ocurrencia de la infección toxoplásmica (Tabla 2), se detectó una asociación significativa entre ellas $\left(\mathrm{Chi}^{2}=6,08 ; \mathrm{P}=0,0137 ; \mathrm{RR}=1,35\right.$ $[1,08<\mathrm{RR}<1,72] ;$ OR $=1,88[1,10<\mathrm{OR}<$ $3,21]$ ). Esto puede deberse a la contaminación del peridomicilio, de las áreas de juego de los niños, con heces de gatos que contienen ooquistes del protozoo. Cabe destacar el rol epidemiológico del gato, que al actuar como huésped definitivo del parásito, es capaz de eliminar hasta 1 millón de ooquistes por gramo de materia fecal, durante 12 semanas $^{26}$. Estos, esporulan entre 1-5 días posteliminación, y pueden permanecer viables durante períodos prolongados (hasta 2 años), de acuerdo a las condiciones del medio ambiente ${ }^{16,26}$.

Al analizar la variable "tenencia de perros" (Tabla 3), se determinó una asociación significativa $\left(\mathrm{Chi}^{2}=13,11 ; \mathrm{P}=0,0029 ; \mathrm{RR}=1,74\right.$ $[1,23<\mathrm{RR}<2,46] ;$ OR $=2,85[1,54<\mathrm{OR}<$ $5,29])$. Esto podría explicarse, al posible rol que juegan los caninos en la transmisión mecánica del parásito, ya que uno de sus hábitos más frecuentes es el de restregarse sobre las heces felinas ${ }^{25}$, contaminando de esta manera, su pelaje con ooquistes infectantes, los cuales pueden ser ingeridos por los niños al jugar con sus mascotas. También se ha demostrado en los perros la existencia de coprofagía de heces de gatos lo que puede permitir la ingesta de ooquistes. Cuando esto sucede, más del $90 \%$ de ellos eclosionan y liberan sus esporozoítos, los cuales invaden nuevos tejidos del animal, pero los restantes
Tabla 4. Serología anti Toxoplasma gondii y tenencia de gatos $y$ perros

\begin{tabular}{lccr}
\hline & \multicolumn{3}{c}{ Serología } \\
& Positivos & Negativos & Total \\
\hline Gatos y Perros & 62 & 35 & 97 \\
Ni gatos / Ni Perros & 10 & 31 & 41 \\
Total & 72 & 66 & 138 \\
\hline
\end{tabular}

Tabla 5. Serología anti Toxoplasma gondii y presencia de gatos en peridomicilios con tierra

\begin{tabular}{lccc}
\hline & \multicolumn{2}{c}{ Serología } & \\
& Positivos & Negativos & Total \\
\hline Tierra c/gatos & 71 & 47 & 118 \\
Tierra s/gatos & 56 & 66 & 122 \\
Total & 106 & 75 & 240 \\
\hline
\end{tabular}

Tabla 6. Serología anti Toxoplasma gondii y presencia de perros en peridomicilios con tierra

\begin{tabular}{lccr}
\hline & \multicolumn{2}{c}{ Serología } & \\
& Positivos & Negativos & Total \\
\hline Tierra c/perros & 101 & 70 & 171 \\
Tierra s/perros & 24 & 45 & 69 \\
Total & 125 & 115 & 240 \\
\hline
\end{tabular}

adquieren importancia epidemiológica, ya que se pueden eliminar en las heces del perro, sin sufrir ningún tipo de modificaciones. Debido a que los perros carecen de la costumbre de enterrar sus deyecciones, se favorece la dispersión de estas formas infectantes, y la consecuente contaminación de las áreas de juego infantiles ${ }^{25}$. A esto se le agrega la particularidad que presentan la mayoría de las viviendas de la zona en estudio, de carecer de cercos perimetrales, por lo que los animales deambulan de una vivienda a otra.

Otro factor de riesgo asociado estadísticamente a la infección, resultó ser la "tenencia conjunta de gatos y perros" (Tabla 4) $\left(\mathrm{Chi}^{2}=18,04 ; \mathrm{P}=\right.$ 0,0000216; RR = 2,62 [1,50<RR < 4,58]; OR = $5,49[2,25<$ OR < 13,67]). En los niños que cohabitaban con gatos, con perros, o con gatos y perros, la serología positiva a $T$. gondii fue del $60,32 \%, 59,78 \%$ y $63,92 \%$, respectivamente; mientras que, tan sólo, un $24,4 \%$ de los que no convivían con ninguno de estos animales, presentaron anticuerpos contra el parásito. En consecuencia, se puede determinar que, la 
presencia de gatos y/o perros son factores de riesgo de importancia.

Al evaluar las condiciones higiénicas domiciliarias de los hogares encuestados, se observó que un $46,6 \%$ de ellos presentaban una adecuada higiene, mientras que en un $53,4 \%$ las condiciones de saneamiento fueron inadecuadas. Por otra parte, al correlacionar esta variable con la correspondiente serología, no se halló asociación significativa entre ellas, por lo que se determinó que no actúa como factor de riesgo $\left(\mathrm{Chi}^{2}=1,60 ; \mathrm{P}=0,2058 ; \mathrm{RR}=0,86[0,68<\mathrm{RR}\right.$ $<1,09]$; OR $=0,72[0,43<$ OR $<1,23])$.

$\mathrm{Si}$ bien las características del peridomicilio (tierra/cemento) no presentaron asociación estadística con la ocurrencia de la infección $\left(\mathrm{Chi}^{2}\right.$ $=0,04 ; \mathrm{P}=0,8378 ; \mathrm{RR}=0,94[0,52<\mathrm{RR}<$ $1,70] ;$ OR $=0,87[0,19<\mathrm{OR}<3,84])$, al relacionar, conjuntamente, las variables "peridomicilio c/tierra"+"tenencia de gatos" (Tabla 5), sí se detectó asociación entre ellas $\left(\mathrm{Chi}^{2}\right.$ $=4,90 ; \mathrm{P}=0,02684 ; \mathrm{RR}=1,31[1,03<\mathrm{RR}<$ 1,67]; OR $=1,78$ [1,03 < OR < 3,07]). Además, de las 118 viviendas que presentaban tierra en sus peridomicilios y en donde se convivía con gatos, un $97,45 \%$ de ellos defecaban en el patio, y tan sólo, un 2,55\% lo hacía en el interior de la misma. Del mismo modo, se halló asociación significativa con la variable "peridomicilio c/ tierra"+"tenencia de perros" ( Tabla 6$),\left(\mathrm{Chi}^{2}=\right.$ 11,$60 ; \mathrm{P}=0,00065 ; \mathrm{RR}=1,70[1,20<\mathrm{RR}<$ $2,40] ;$ OR $=2,71[1,46<$ OR $<5,05])$. Estas asociaciones están corroborando lo descripto anteriormente, o sea que si bien la presencia de gatos y perros es significativa en la transmisión del parásito, no tendrían mayor trascendencia sin la presencia de un medio capaz de contener los estados infectantes del mismo, como lo es el suelo (tierra). Hechos similares se han observado en Panamá ${ }^{17}$ y en Costa Rica ${ }^{16}$.

En el análisis de la variable "verduras crudas", se advirtió que en un 79,52\% de los 249 hogares encuestados, se consumía verduras de huerta adecuadamente lavadas (con agua potable y gotas de vinagre o de lavandina), tan sólo un 1,6\% lo hacía sin ningún tipo de tratamiento previo, mientras que el $18,88 \%$ no consumía este tipo de alimento. De tal manera, que no se halló asociación estadística entre el consumo de verduras crudas y la infección $\left(\mathrm{Chi}^{2}=0,98 ; \mathrm{P}=\right.$ 0,$3221 ; \mathrm{RR}=1,50[0,84<\mathrm{RR}<2,69] ; \mathrm{OR}=$ $3,00[0,27<$ OR $<76,16])$. Cabe destacar que a pesar de las bajas condiciones socioeconómicas de la zona, todas las viviendas contaban con servicio de agua potable, ya sea de red, o bien de pico público.

En cuanto, al "consumo de carnes", se observó que un $83,53 \%$ de la población ingería carne bien cocida, y un $16,47 \%$ la prefería semicocida, y no se encontraron hogares en que se consumiera la carne totalmente cruda. Esta variable determino que el consumo de carne cocida" es un factor de protección contra el riesgo de contraer la infección $\left(\mathrm{Chi}^{2}=4,84 ; \mathrm{P}=\right.$ 0,$02778 ; \mathrm{RR}=0,73[0,56<\mathrm{RR}<0,93] ; \mathrm{OR}=$ $0,46[0,21<\mathrm{OR}<0,98])$. Esto se fundamenta en el hecho de que los quistes tisulares presentes en carnes de consumo, no son afectados por agentes físicos, fármacos ${ }^{5}$, ni por la acción de las microondas; pero sí pueden ser inactivados térmicamente a $67^{\circ} \mathrm{C}(27)$. Además, el 99,2\% de la población consumía, preferentemente, carne de bovinos; cabe destacar que esta especie se considera poco receptiva a la infección, siendo capaz de eliminar rápidamente al protozoo, tras la fase de parasitemia, o de reducir marcadamente su número en los tejidos, por lo que se puede inferir que la persistencia de $T$. gondii en carnes de bovinos, es probablemente irrelevante ${ }^{28}$.

\section{RESUMEN}

El presente estudio se realizó en el marco de un programa interinstitucional entre la Universidad Nacional de Río Cuarto y la Municipalidad de dicha ciudad, donde se trabajó conjuntamente con Efectores de Salud, ubicados en la zona de estudio. El área analizada se encuentra situada dentro del radio urbano de la ciudad, y se extiende en ambas márgenes del río Cuarto. El objetivo principal del trabajo fue determinar la seroprevalencia a $T$. gondii, en la población infantil de 0 - 15 años, presente en Áreas de Riesgo de la ciudad de Río Cuarto, y analizar e interpretar aquellas variables que interactuan en la presentación de esta zoonosis; ya que en nuestra región no existen datos que revelen la magnitud de la situación actual. El estudio realizado durante los años 1999-2001, fue de corte transversal, estimando el muestreo en 257 niños, realizándose un screening de 1/16 mediante la técnica de Inmunofluorescencia Indirecta. Se confeccionaron encuestas epidemiológicas que permitieron establecer el grado de riesgo de algunos factores 
geo-bio-ambientales involucrados en la infección. Se encontró una eroprevalencia del $51,75 \%$; No se observaron diferencias significativas de acuerdo al sexo; sí se observó un aumento de la prevalencia con respecto a la edad. Se determinaron como factores de riesgo, asociados a la ocurrencia de la infección toxoplásmica, la convivencia con gatos y/o perros, especialmente en aquellas viviendas cuyos peridomicilios contaban con la presencia de tierra (jardines, corrales, huertas, gallineros). A través del presente trabajo se ha determinado, como principal fuente de transmisión, para los niños provenientes de la zona estudiada, el contacto con el medio ambiente contaminado con ooquistes de $T$. gondii. Esta información le permitirá a la Municipalidad el abordaje de la problemática existente, formulando programas específicos para el control de dicha zoonosis.

\section{REFERENCIAS}

1.- ACHA P, SZYFRES B. Zoonosis y enfermedades transmisibles comunes al hombre y a los animales. Publicación Científica $\mathrm{N}^{\circ}$ 503. 2da Edición. Organización Panamericana de la Salud, Organización Mundial de la Salud. 1992.

2.- ATIAS A. Parasitología Clínica. Santiago. Chile. Editorial Mediterráneo. 1995. 615 p.

3.- FRENKEL J K. Citado por Jacobs I. New knowledge of Toxoplasma and toxoplasmosis. Adv Parasitol 1973; 11: 631-69.

4.- VIENS P, AUGER P, VILLENEUVER, STEFANESCUSOURCE I. Serological survey for congenital toxoplasmosis among 4.136 pregnant women. Trans Roy Soc Trop Med Hyg 1977; 71: 136-9.

5.- PIZZI H. Toxoplasmosis. 1ra Edición. Rhone Poulenc Rorer Argentina 1997.

6.- DUBEY J P. Epidemiology of toxoplasmosis in developed and developing countries. Parasitol al Día 1995; 19 (123-C): 154.

7.- VAZQUEZ M, AMBROGIO A, CHIARETTA A et al. Estudio de la toxoplasmosis en mujeres embarazadas, en la zona de Río Cuarto. II Jornadas Científico Técnicas, Facultad de Agronomía y Veterinaria. Universidad Nacional de Río Cuarto. Córdoba 1987.

8.- CENSO NACIONAL DE POBLACIÓN Y VIVIENDA. 1991.

9.- DIVISIÓN ZOONOSIS MUNICIPALIDAD DE RÍO CUARTO. 1996.

10.- WAISSMAN I, LARRIESTRA A, GONZÁLEZ J et al. Desarrollo de un sistema de información geográfica aplicada al estudio de la mortalidad infantil en la Ciudad de Río Cuarto, Córdoba. Argentina. IV Jornadas Científico - Técnicas. Facultad de Agronomía y Veterinaria. Universidad Nacional de Río Cuarto. Río Cuarto. Córdoba 1997.

11.- WEYERS A, CHASSAGNADE M, ESPOSITO N,
UGNIA L. Encuesta Censal Canina y Felina. Río Cuarto, Córdoba. IV Jornadas Científico - Técnicas, Facultad de Agronomía y Veterinaria. Universidad Nacional de Río Cuarto. Córdoba 1997.

12.- THRUSFIELD M. Epidemiología Veterinaria. 1ra Edición. Editorial Acribia S.A. 1990.

13.- BEAUVAIS B, GARIN Y, LANGUILLAT G, LARIVIERE M. La toxoplasmose au Gabon Oriental. Resultats d'une enquete serologique. Bull Soc Path Exot 1978; 71: 172-81.

14.- GORODNER J O. Situación de toxoplasmosis en Argentina y el Mundo. Jornadas de Actualización de toxoplasmosis. Mendoza. Argentina 2000.

15.- FAYER R. Toxoplasmosis Update and Public Health Implications. Can Vet J 1981; 22: 344-52.

16.- FRENKEL J K, RUIZ R. Human toxoplasmosis and cat contact in Costa Rica. Amer Soc Trop Med Hyg 1980; 29: 1167-80.

17.- ETHEREDGE G D, FRENKEL J K. Human Toxoplasma infection in Kuna and Embera children in The Bayano and San Blas, eastern Panamá. Amer Soc Trop Med Hyg 1995; 53: 448-57.

18.- RICCIARDI I D, SABROZA P C, SANDOVAL E D, MAYRINK B W. Seroepidemiological study on the prevalence of human toxoplasmosis in Brazil. Rev Microbiol (S Paulo) 1978; 9: 181-7.

19.- VELASCO-CASTREJON O, SALVATIERRA-IZABA B, VALDESPINO J L et al. Seroepidemiología de la toxoplasmosis en México. Salud Pública en México 1992; 34: 222-9.

20.- ROMAÑA C, LIFSCHITZ J. Citado por Bergoglio R. Toxoplasmosis, algunas consideraciones. Rev Infectol Córdoba 1974; 1: 5-23.

21.- ROMAÑA C. Citado por Gorodner JO. Situación de toxoplasmosis en Argentina y el Mundo. Jornadas de Actualización de Toxoplasmosis. Mendoza. Argentina. 2000.

22.- AVERBACH. Citado por Gorodner JO. Situación de toxoplasmosis en Argentina y el Mundo. Jornadas de Actualización de Toxoplasmosis. Mendoza. Argentina. 2000.

23.- PIZZI H, BENVISSUTO G, NAVARROZ et al. Estudio serológico para toxoplasmosis en dadores de sangre del Hospital Pediátrico de Córdoba, Argentina. Rev Fundación Argentina para el Estudio de la Toxoplasmosis (FAPET), Bs. As. 1983; 1: 3-4.

24.- LUPO J, ANTOLIN M. Índice serológico de toxoplasmosis en población general de Santa Fe. Argentina. Rev. Fundación Argentina para el Estudio de la Toxoplasmosis (FAPET), Bs. As. 1984; 2: 9.

25.- FRENKEL J K, LINDSAY D S, PARKER B B. El supuesto rol de perros en la transmisión mecánica de la toxoplasmosis. Parasitol Día 1995; 19 (137-C): 154.

26.- DUBEY J P. Toxoplasma gondii oocyst survival under defined temperatues. J Parasitol 1998; 84: 862-5.

27.- LUNDEN A, UGGLA A. Infectivity of Toxoplasma gondii in mutton following curing, smoking, freezing or microwaving cooking. Inst J Food Microbiol 1992; 15: 357-63.

28.- DUBEY J P. Isolation of Toxoplasma gondii from a Naturally Infected Beef Cow. J Parasitol 1992; 78: 151-3. 